\title{
A.c. stability and a.c. loss in composite superconductors*
}

\author{
L.J.M. van de Klundert \\ University of Twente, Department of Applied Physics, PO Box 217, 7500 AE Enschede, \\ The Netherlands \\ Two methods of loss calculations are reviewed. The first method, for loss calculations in \\ wires, uses a numerical solution of the Maxwell equations. The second method makes use \\ of Kirchhoff's equations and is much better suited to cables, including braids. Both \\ methods require a knowledge of the constitutive equations relating $E$ and $j$ or $V$ and $I$ in \\ the composite conductor. Experimental results regarding the stability of large cables are \\ presented and a way of improving the stability of a single strand is suggested.
}

Keywords: superconductors; magnet stability; a.c. losses

The occurrence of a quench in a large superconducting system leads to total dumping of the stored energy and restarting of the cooling procedures before the system can be put into operation again. The strategy used to prevent quenches is different for various systems. Globally we can distinguish three kinds of magnet systems with reference to the time dependence of the current and the fields applied to the conductor.

1 Both current and field are time independent; this is the well known d.c. case.

2 The current is constant but the applied field is time dependent; for instance, the toroidal coils of a tokamak experience the a.c. field of the ohmic heating coils and the poloidal field coils.

3 The current through the magnet has a (periodic) time dependence. Then the field applied to each individual strand will also be time dependent. Examples can be found in many power applications, such as generators, transformers, etc.

In the first two cases the strategy is based on a number of methods, such as increasing the heat capacity and normal conductivity by adding stabilizing materials such as copper $(\mathrm{Cu})$ or aluminium $(\mathrm{Al})$ outside the superconducting filament region, or by increasing the contact area with the (superfluid) coolant, as in cables in conduit. Much attention, however, has to be paid to heat generation caused by wire motion or heat generated in the conductor by coupling and magnetization currents, as well as by the transport current itself. By magnetization currents we mean those currents that have closed loops within one filament, whereas coupling currents

* Paper presented at the Symposium on Superconductor Stability, 13- 15 November 1990, Yokohama, Japan

$0011-2275 / 91 / 070612-06$

(c) 1991 Butterworth - Heinemann Ltd

612 Cryogenics $1991 \mathrm{Vol} 31$ July may have closed loops either within the entire strand or in the cable. Usually, the latter decay with a much larger time constant. Since the loop of the coupling currents exists partly within the matrix and partly within the superconducting filaments the transport current may compete with the magnetization and the coupling currents within each filament. An extra voltage is then required to maintain the transport current and the superconductor has an apparent resistance due to the applied time dependent field.

The prevention of a quench in the third case, however, cannot be based on the addition of a stabilizing material outside the filaments, since the power dissipated by the coupling currents would then increase accordingly and, moreover, degradation of the transport current would result. It may be noted that by addition of a stabilizing material a very low ohmic parallel path for the transport current is also created in case the critical current density is exceeded locally. Apart from preventing local burn out of the wires, complete restoration of the fully superconducting state can also be achieved.

In the next section general methods of calculating the loss in composite conductors will be considered and experimental results concerning the stability of cables will be treated. An old concept concerning improvement of the stability of wires and cables in an a.c. transport current regime will be discussed.

\section{Loss calculations}

Loss calculations in wires will be dealt with first and methods for cables will be shown in the second part of this section. The a.c. loss theory for wires has been developed by several authors ${ }^{1-3}$. A general method for wires, based on solving the Maxwell equations, was introduced by $\mathrm{Rem}^{4}$. In a cylindrical coordinate system 
$(r, \phi, z), \nabla \times E=\dot{B}$ has the components

$\frac{1}{r} \partial_{\varphi} E_{z}-\partial_{z} E_{\varphi}=-\dot{B}_{r}$

$\partial_{z} E_{r}-\partial_{r} E_{z}=-\dot{B}_{\varphi}$

$\frac{1}{r}\left(\partial_{r} r E_{\varphi}-\partial_{\varphi} E_{r}\right)=-\dot{B}$

It is useful to split the local magnetic field rate, $\dot{B}$, into an externally applied component, $\dot{B}^{\mathrm{A}}$, and the induced component, $\dot{B}^{I}$. If $\dot{B}=0$ the solution to the above set of equations is called the stationary solution. The solution also has to obey

$\nabla \cdot j=0$

The solution depends strongly on the chosen constitutive equation relating $E$ and $j$. The components are

$j_{r}=\sigma_{r r} E_{r}=\sigma_{\perp} E_{r}$

$j_{\varphi}=j^{\mathrm{s}} \sin \psi+\sigma_{\varphi \varphi} E_{\varphi}+\sigma_{\varphi z} E_{z}$

$j_{z}=j^{\mathrm{s}} \cos \psi+\sigma_{\varphi z} E_{\varphi}+\sigma_{z z} E_{z}$

In these equations $j^{s}$ is the superconducting current in the filament direction and $\sigma_{\perp}$ is the conductivity perpendicular to the filaments. If we denote the conductivity parallel to the filaments by $\sigma_{\|}$then

$\sigma_{\varphi \varphi}=\sigma_{\perp} \cos ^{2} \psi+\sigma_{\|} \sin ^{2} \psi$

$\sigma_{\varphi z}=\left(\sigma_{\|}-\sigma_{\perp}\right) \sin \psi \cos \psi$

$\sigma_{z z}=\sigma_{\|} \cos ^{2} \psi+\sigma_{\perp} \sin ^{2} \psi$

where $\psi=\operatorname{arctg}\left(2 \pi r / L_{\mathrm{p}}\right)$ and is the twist angle.

The relation between $j$ s and $E_{\|}=E_{z} \cos \psi+E_{\varphi} \sin \psi$ is given by

$j^{s}=j_{\mathrm{c}} E_{\|} / \epsilon \quad$ if $\quad\left|E_{\|}\right|<\epsilon$

$j^{\mathrm{s}}=j_{\mathrm{c}} \operatorname{sign}\left(E_{\|}\right)$if $\quad\left|E_{\|}\right| \geq \epsilon$

$\epsilon=\frac{8}{3 \pi} R_{\mathrm{f}} \partial_{\mathrm{t}}\left(B_{r}^{2}+B_{\varphi}^{2}\right)^{1 / 2}$

where $R_{\mathrm{f}}$ is the radius of the filament.

The value of the loss power density can be calculated according to $p=E \cdot j-M \cdot \dot{B}$ and integrating over the volume of the wire. The first term represents the contribution of coupling and transport currents and the second the contribution of the magnetization currenis.

With appropriate boundary conditions the loss power density can be found for wires of finite and infinite length ${ }^{5}$. Simple analytical approximations exist when $\dot{B}^{\mathrm{A}}$ is constant and has only one component, either perpendicular or parallel to the wire axis. Approximate solutions also exist when $\dot{B}_{+}$and $\dot{B}_{\|}$are periodic functions along the directions of the wire axis. The applied field has to obey $\nabla \cdot B^{A}=0$ and $\nabla \times B^{A}=0$.

For non-stationary solutions the sets of equations $\nabla \cdot B^{\mathrm{I}}=0$ and $\nabla \times B^{\mathrm{I}}=\mu_{0} j$ also have to be solved simultaneously. This can only be done numerically. The combination of spatially inhomogeneous applied fields and non-stationary behaviour, however, requires so much computer time that so far no solutions have been obtained. The main results can be given as follows

1 The loss power density is a non-uniform function of position. The larger values exist in the saturated parts: $\left|E_{\|}\right| \geq \epsilon$.

2 The parts saturated by coupling currents cannot contribute to the transport current. As an example, consider the maximum transport current for a perpendicular field rate, $\dot{B}_{\perp}$, which is reduced accordingly to

$$
I_{\max }=I_{\mathrm{c}}\left[1-\frac{\sigma_{\perp} R_{\mathrm{w}}\left|\dot{B}_{\perp}\right|}{\eta j_{\mathrm{c}}}\left(\frac{L_{\mathrm{p}}}{2 \pi R_{\mathrm{w}}}\right)^{2}\right]
$$

Similar relations hold for spatially periodic values of $\dot{B}_{\|}$.

The loss in hollow twisted cables can also be calculated in a similar way as shown by Hartman ${ }^{5}$. Since no current flows in the $r$-direction an essentially two-dimensional problem has to be solved. Here also stationary and non-stationary solutions can be obtained. Note that for spatially periodic fields no solution exists in the stationary case if the period length of the field, $L_{\mathrm{F}}$, equals the period length of the cable ${ }^{6}$.

Three-dimensional cables, either twisted or braided, can be modelled with three-dimensional networks ${ }^{7}$. In Figure $I$ an example is given of a network representing
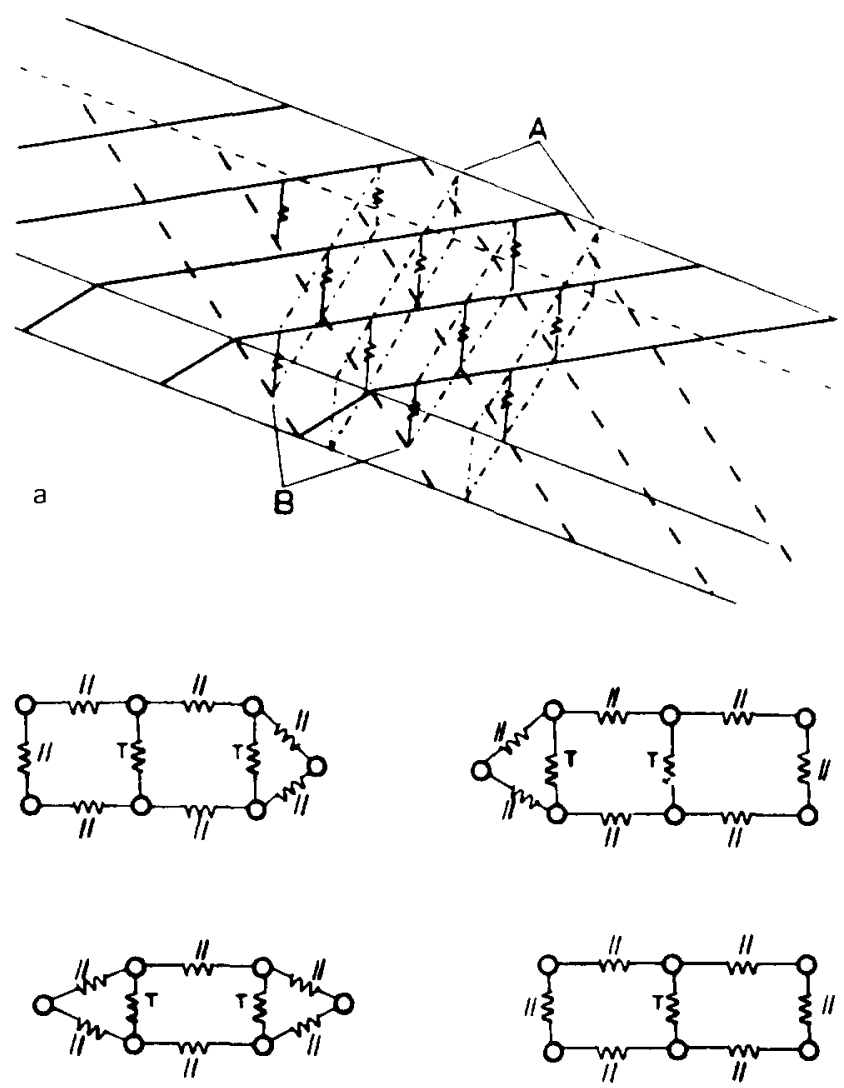

b

Figure 1 (a) Schematic view of the Rutherford cable showing the two types of cross-sections, $A$ and $B$. The periodicity of the cable is $L_{c} / N$. (b) Meshes in a place perpendicular to the cable for an even number of strands (upper figure, 6) and for an odd number of strands (lower figure, 7) 
a Rutherford cable. The Kirchoff laws

$\sum_{i} I_{i}=0$

and

$$
\sum_{j} V_{j}=-V_{\text {ind }}
$$

are equivalent to the Maxwell equations used in the case of the wire. The stationary and non-stationary solutions are obtained by calculating $V_{\text {ind }}$ with the help of $\dot{B}^{\mathrm{A}}$ or using $\dot{B}^{\mathrm{A}}+\dot{B}^{\mathrm{I}}$, respectively. The latter is a much more complicated procedure, as in the case of the wire, where the equations $\nabla \cdot B^{\mathrm{I}}=0$ and $\nabla \times B^{\mathrm{I}}=\mu_{0} j$ had to be solved.

The solution method requires constitutive equations. Three forms have to be used:

(a) normal currents in the contact points between strands

$$
V_{\mathrm{n}}=R_{\mathrm{n}} I_{\mathrm{n}}
$$

(b) unsaturated superconducting currents, $I_{u}$

$$
V=I_{\mathrm{u}} /\left(I_{\max } / V_{0}+1 / R_{\mathrm{p}}\right) \quad \text { if } \quad|V|<V_{0}
$$

where: $V_{0}$ is the saturation threshold, $V_{0}=$ $8 R_{\mathrm{f}} L_{\mathrm{e}}\left|\dot{B}_{\perp}\right| / 3 \pi ; L_{\mathrm{e}}$ is the length of the elements of the strand; $R_{\mathrm{p}}$ is the parallel resistance of these elements; and $I_{\max }$ is as defined above; and

(c) saturated superconducting currents, $I_{\mathrm{s}}$

$$
V=R_{\mathrm{p}}\left[I_{\mathrm{s}}-I_{\max } \operatorname{sign}(V)\right]
$$

The non-linear behaviour of the constitutive equations implies that the solution, in all cases mentioned above, can be found only in an iterative way. A typical result ${ }^{8}$ for the 29-strand NET braid is depicted in Figures 2 and 3 , which show the currents in the contact resistances as well as the order of the positions where the strands saturate when increasing $\left|\dot{B}_{\perp}\right|$. In Figure 2 a uniform

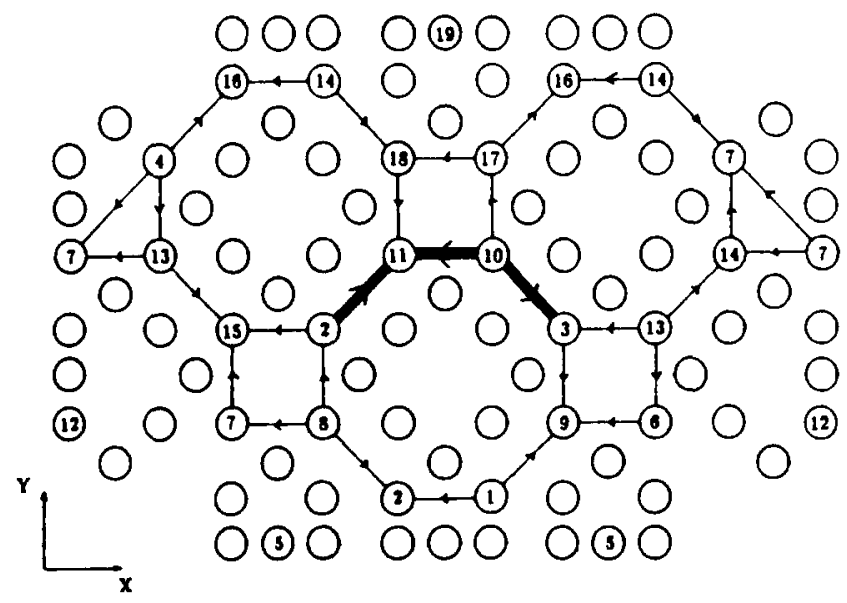

Figure 2 Saturation process for $d B_{x} / d t$ of $1 \mathrm{~T} \mathrm{~s}^{-1}$ with increasing 10 . The numbers indicate the order of the positions where the strands saturate

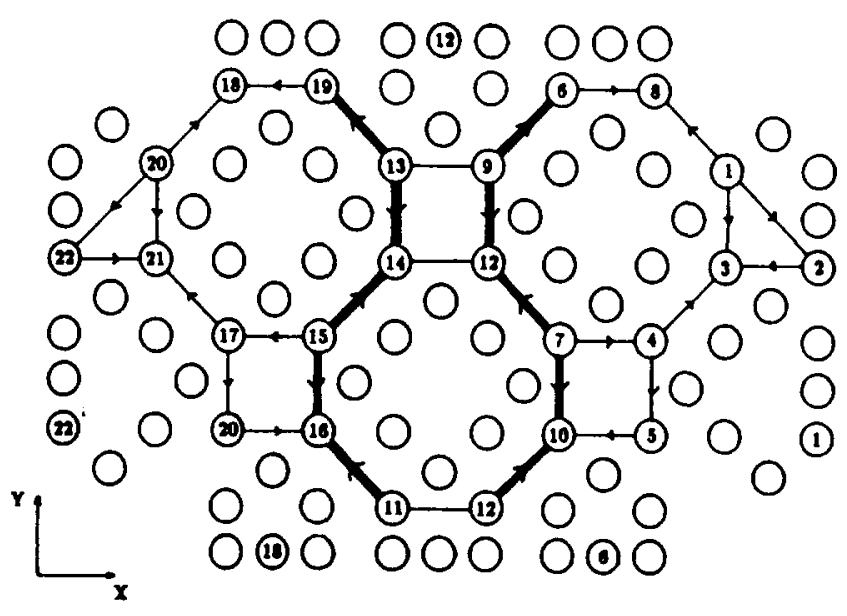

Figure 3 Saturation process for $\mathrm{d} B_{\gamma} / \mathrm{dt}$ of $1 \mathrm{~T} \mathrm{~s}^{-1}$ with increasing $I_{0}$. The numbers indicate the order of the positions where the strands saturate

$\dot{B}_{\perp}$ is applied parallel to the wide side of the braid (the $x$-direction); in Figure 3 it is perpendicular to the wide side (the $y$-direction). Figure 4 shows the total loss power density as a function of $L_{\mathrm{F}} / L_{\mathrm{C}}$ in the stationary case for an applied field in the $x$-direction.

In this section it has been shown that the loss in a complicated composite conductor can be modelled. Difficulties arise from the exact form of the non-linear constitutive equations. The calculations take much time because of their iterative nature, especially when nonstationary solutions are required. Also spatially inhomogeneous applied field rates complicate the calculations. In conjunction with the cooling system available, however, the wire and cable design can be such that safe operation can be assumed.

\section{Unstable behaviour of cables}

Whenever an instability occurs in a single wire, either through a flux jump or any other kind of heat production

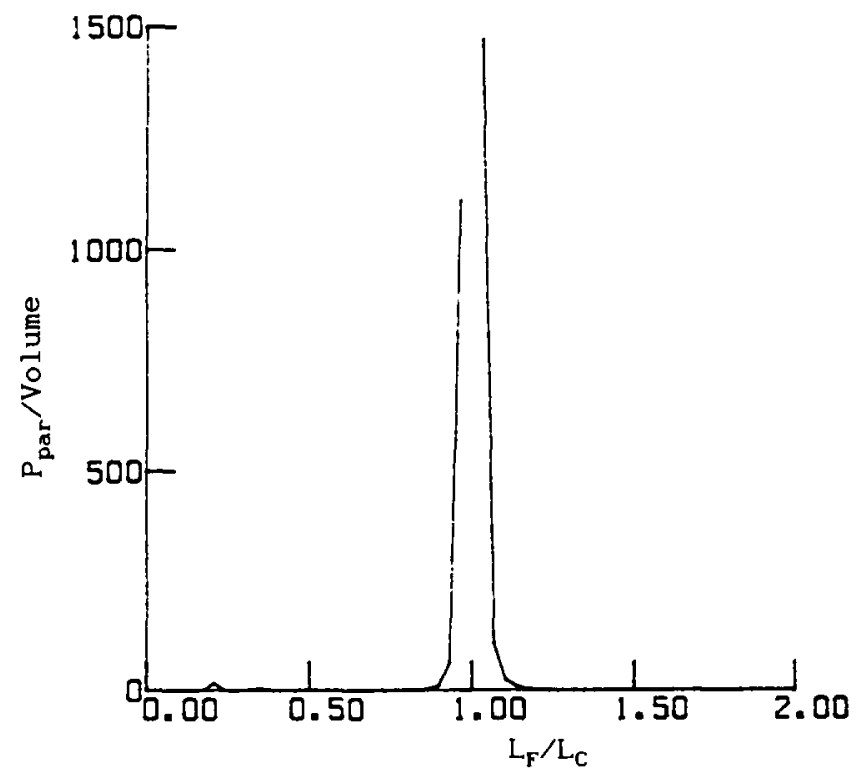

Figure 4 Total loss power density, $P_{\text {in }}$ /volume (in $\mathrm{W} \mathrm{m}^{-3}$ ) as a function of $L_{F} / L_{C}$ for the 29-strand braid exposed to a changing field in the $x$-direction 

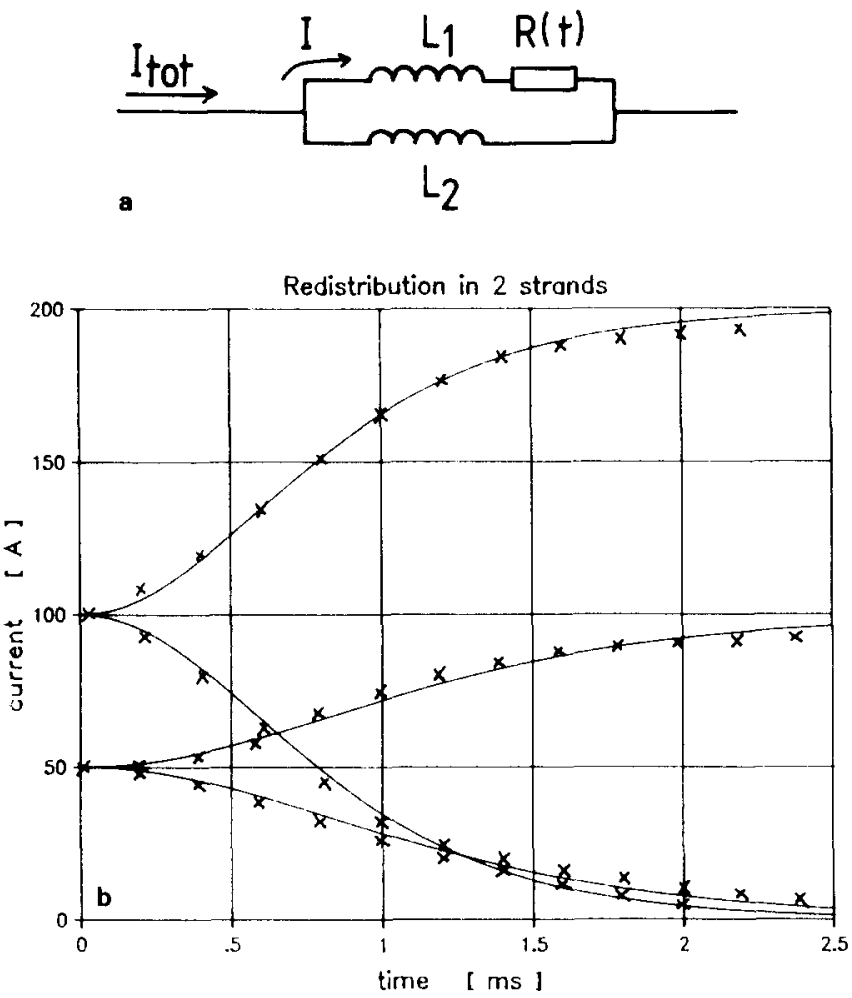

Figure 5 (a) Schematic view of the two-strand circuit. (b) Time dependence of the currents in each strand: $X$, experimental measurements; —_ calculated values

such as that due to wire motion, a quench propagates along the wire with a velocity typically of the order of $10-100 \mathrm{~m} \mathrm{~s}^{-1}$. This has been observed in $\mathrm{Cu}$ matrix and $\mathrm{Cu}-\mathrm{Ni}$ matrix wires ${ }^{9}$.

In a multi-strand system or cable a totally different behaviour pattern exists. This can be shown by the following example of a two-strand cable ${ }^{10}$ (see Figure $5 a$ ). Suppose in one strand carrying a current $I$ some heat is introduced to initiate a quench; a resistance $R(t)$ is then built up. To a good approximation, the quench velocity is proportional to the actual current, so that

$\frac{\mathrm{d} R}{\mathrm{~d} t}=a I$

The current $I$ has to be transferred to the other strand. As long as the total current remains constant we may write $L I=R(t) I=0$, where $L$ is the effective inductance of the loop and $L=L_{1}+L_{2}-2 M_{12}$. Then the following equation holds for $I$

$\ddot{I}-\dot{I}^{2}+b I^{3}=0, \quad b=a / L$

which has the solution

$I(t)=I_{0} / \cosh ^{2}\left[t\left(b I_{0} / 2\right)^{1 / 2}\right]$

where $I_{0}$ is the initial value of $I$.

The solution for $I(t)$ is shown in Figure $5 b$ for several values of $I_{0}$, together with the experimental data. The current transfer occurs in a few milliseconds for this $\mathrm{Cu}-\mathrm{Ni}$ wire. As long as the other wire can carry all the current no quench of the total system occurs and the cur- rent may finally redistribute over both wires again. If the total current exceeds some critical level, redistribution will lead to a quench of both strands but now with an apparent quench velocity much higher than the usual one because the critical current is exceeded everywhere. The same phenomenon occurs at different current levels in twisted cables of five or six strands ${ }^{11,12}$. If this twisted cable is used as a subcable of a larger cable able to carry up to $100 \mathrm{kA}$ this may lead to a total quench.

The redistribution of the current between subcables is much more difficult because almost no current can flow through the contact points between the subcables. This was illustrated very clearly by the experiments of Mulder $^{12}$ where two cables, one with $\mathrm{Cu}$ matrix strands, the other with a $\mathrm{Cu}-\mathrm{Ni}$ matrix, were used for stability experiments. Figures $6-9$ show that the choice

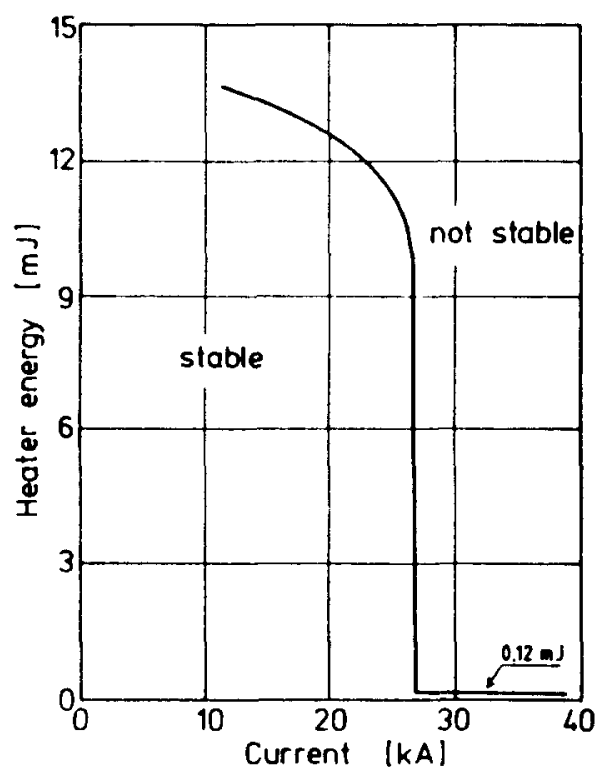

Figure 6 Quench sensitivity of an 864-strand cable with $\mathrm{Cu}$ - Ni matrix

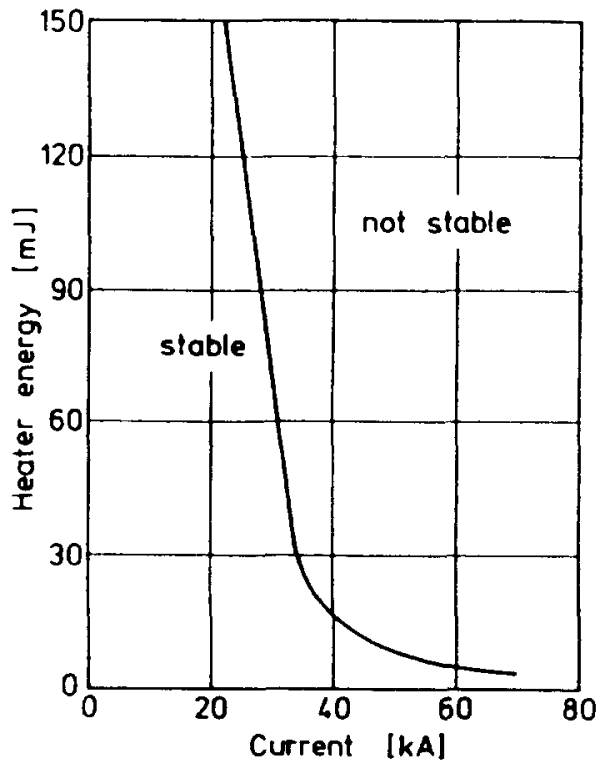

Figure 7 Quench sensitivity of a 1008-strand cable with a $\mathrm{Cu}$ matrix 


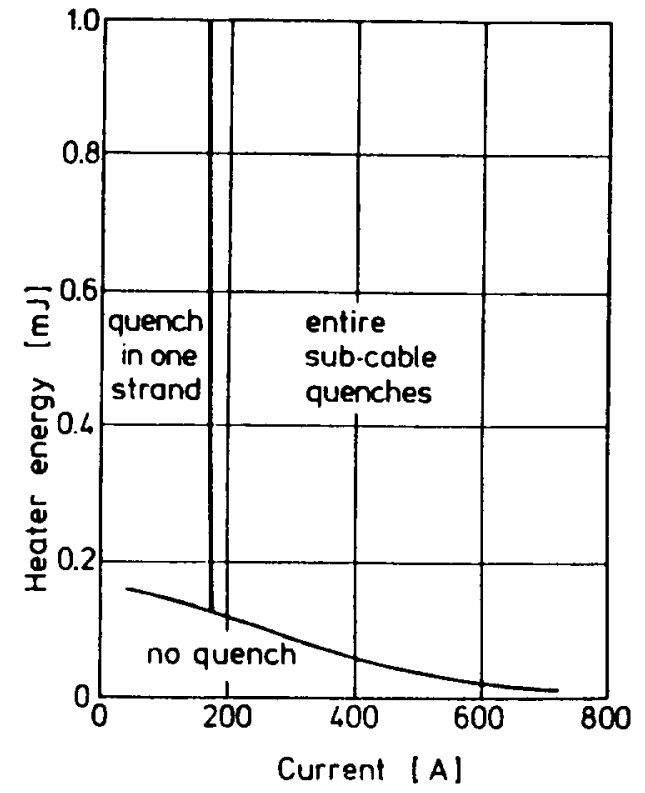

Figure 8 Quench sensitivity of a six-strand subcable with a $\mathrm{Cu}-\mathrm{Ni}$ matrix

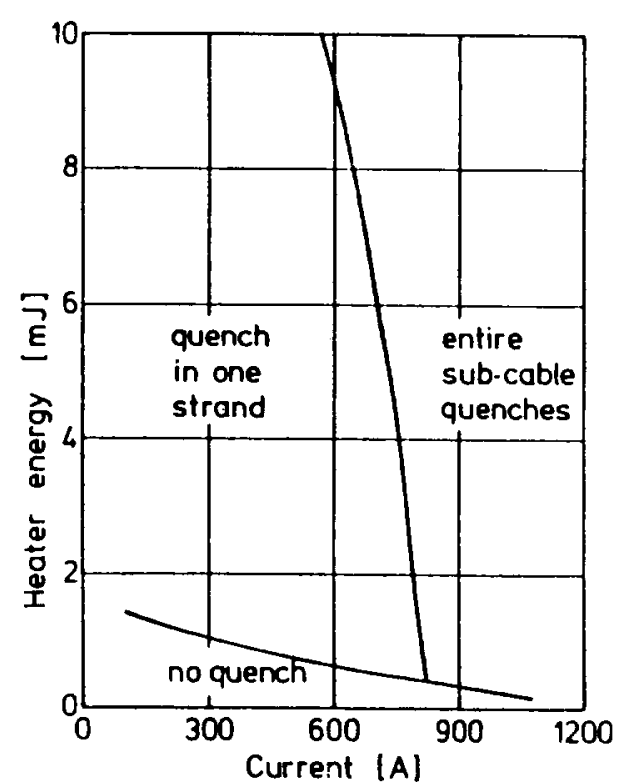

Figure 9 Quench sensitivity of a seven-strand subcable with a Cu matrix

of the matrix material only has influence on the stability margin, i.e. on the magnitude of the heat pulse required to quench one subcable. Qualitatively, the phenomenon of redistribution is the same in the $\mathrm{Cu}$ and the $\mathrm{Cu}-\mathrm{Ni}$ cables.

\section{Improvement of the stability of a wire}

In the previous sections it has been shown that current redistribution in a strand due to time dependent applied fields causes heat dissipation but does not necessarily lead to a quench. If, however, one strand in a cable quenches due to some internal or external cause, the sub-

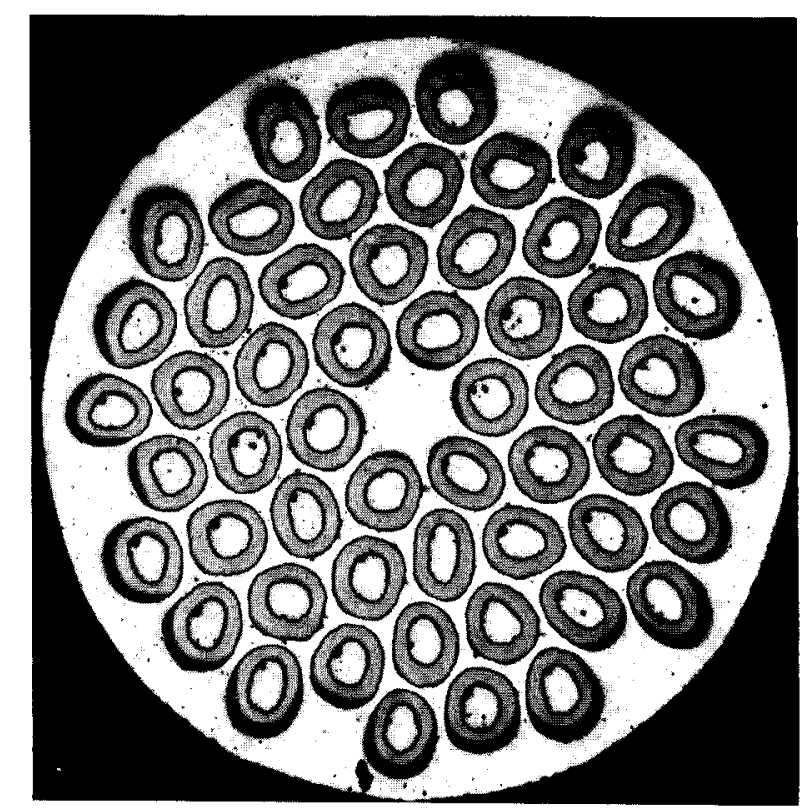

Figure 10 Photograph of cross-section of a wire having filamentary tubes with highly conductive material (Al) inside them

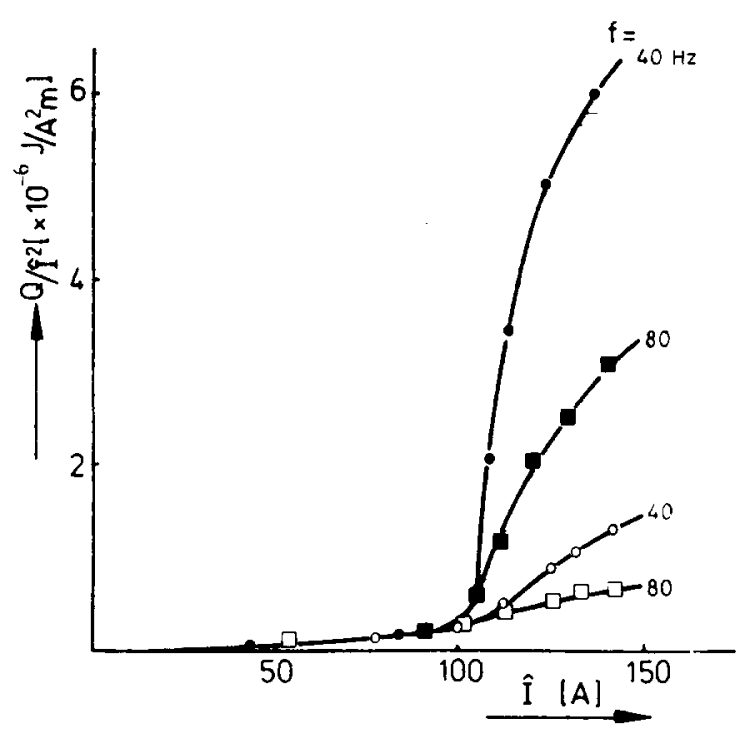

Figure 11 Measured transport current loss of a wire with an inner core of $\mathrm{Al}$ inside the filament. The loss versus current amplitude is shown for two frequencies, 40 and $80 \mathrm{~Hz}$. In the two lower cases an extra $\mathrm{Cu}$ layer is present

cable and the whole cable may quench because current distribution in the cable cannot be achieved in a short time interval, say $<1 \mathrm{~ms}$. The addition of stabilizing material outside the filament that can also carry some of the excess current does not help for this short time-scale. However, addition of stabilizing material inside the filament ${ }^{13,14}$, such as very high conductivity $\mathrm{Al}$ (see Figure 10 ) has proved capable of enabling a.c. currents at frequencies up to $200 \mathrm{~Hz}$ to flow without problems. In this case no extra coupling currents are introduced in the low conductivity matrix.

The filament diameter of these hollow filaments may be a few micrometres, introducing a relatively large magnetization loss ${ }^{15}$, but the cooling problem is 
believed to be of less importance as long as the stability of each individual strand in a cable for power frequency use is guaranteed by the proper position of the stabilizing material.

Figure 11 shows the transport current loss of such a wire having an $\mathrm{Al}$ core inside the filament. The critical current of this wire is $100 \mathrm{~A}$ but, as seen in Figure 11 , it is possible to introduce current amplitudes much larger than the critical current.

\section{Conclusions}

It has been shown that for loss calculations two methods can be used, both of which are based on numerical schemes. One uses the Maxwell equations and is very well suited for wires. The other uses Kirchhoff's equations and is particularly suited to cables of any type. Both systems of equations require adequately formulated constitutive equations describing the local non-linear $E-j$ or $V-I$ relations.

The network method is also very well suited to describing the quench behaviour of cables. Experiments have shown that the quench of one strand can have severe consequences for the whole cable. Future research for a.c. applications has to be directed towards preventing quenches in strands rather than just reducing the generated heat. It is suggested that putting a stabilizing high conductivity material such as $\mathrm{Al}$ inside each filament may be a solution. Experimental evidence supporting this solution has been shown.

\section{References}

1 Carr Jr, W.J. AC loss in a twisted filamentary superconducting wire: I and II J Appl Phys (1974) 45929 and 935

2 Morgan, G.H. J Appl Phys (1970) 413673

3 Ries, G. AC-losses in multifilamentary superconductors at technical frequencies IEEE Trans Magn (1977) MAG-13 524

4 Rem, P.C. Numerical models for AC superconductor PhD Thesis University of Twente, The Netherlands (1986)

5 Hartmann, R.A. A contribution to the understanding of AC losses in composite superconductors $P h D$ Thesis University of Twente, The Netherlands (1989)

6 Takacs, S. Coupling losses in spatially changing AC fields Cryogenics (1982) 22661

7 Niessen, E.M.J., ter Avest, D. and van de Klundert, L.J.M. Application of the network method to superconductor cables (paper 05.4) Proc LTEC 90 Southampton, UK (1990)

8 Niessen, E.M.J. and van de Klundert, L.J.M. A numerical model for the 29-strand NET braid IEEE Trans Magn (1991) MAG-13 1296

9 ten Kate, H.H.J., Boschman, H. and van de Klundert, L.J.M. Normal zone propagation velocities in superconductivity wires $A d v$ Cryog Eng (1988) 341049

10 Vysotski, V.S. (on leave from the Lebedev Institute, Moscow in Twente, The Netherlands) personal communication (September 1990)

11 van Overbeeke, F. On the application of superconductors in power transformers PhD Thesis University of Twente, The Netherlands (1986)

12 Mulder, G.B.J., Krooshoop, H.J.G., Nijhuis, A., ten Kate, H.H.J. and van de Klundert, L.J.M. A study of quench current and stability of high current multi-strand cables having a $\mathrm{Cu}$ or a $\mathrm{CuNi}$ matrix Adv Cryog Eng (1990) 36279

13 Wagner, G.R., Walker, M.S., Koop, D.A. and Whatstone, C.N. $\mathrm{AC}$ losses and critical current in an aluminium stabilized mixed matrix $\mathrm{NbTi}$ superconductor composite IEEE Trans Magn (1979) MAG-13 217

14 de Reuver, J.L. AC losses in current carrying superconductors $P h D$ Thesis University of Twente, The Netherlands (1985)

15 Eikelboom, J.A. and van de Klundert, L.J.M. Hysteresis losses in hollow superconducting filaments and in a multifilament system Cryogenics (1991) 31363 